\title{
FUNÇÕES DE PEDOTRANSFERÊNCIA PARA A CURVA DE RESISTÊNCIA DO SOLO À PENETRAÇÃO ${ }^{(1)}$
}

\author{
Cinara Xavier de Almeida ${ }^{(2)}$, José Frederico Centurion ${ }^{(3)}$, Onã da \\ Silva Freddi ${ }^{(4)}$, Ricardo Falqueto Jorge ${ }^{(5)}$ \& José Carlos Barbosa ${ }^{(6)}$
}

\begin{abstract}
RESUMO
A estimativa da curva de resistência do solo à penetração (CRP) a partir de variáveis de fácil obtenção, como o conteúdo de água, representa uma medida útil não só para a quantificação do estado de compactação, mas também para facilitar a interpretação da resistência do solo à penetração obtida em diferentes condições de campo. O objetivo deste trabalho foi estimar a CRP em solos de diferentes granulometrias e densidades, a partir de dados obtidos com o penetrômetro de impacto. O experimento foi realizado no laboratório de Pedologia da Faculdade de Ciências Agrárias e Veterinárias (UNESP), Jaboticabal, SP. Foram utilizadas quatro classes de solos: Neossolo Quartzarênico, Argissolo Vermelho-Amarelo, Latossolo Vermelho distrófico e Latossolo Vermelho acriférrico, os quais foram coletados na camada de 0-0,20 m. Colunas de PVC, com dimensões de 0,25 $\mathrm{m}$ de diâmetro e $0,6 \mathrm{~m}$ de altura, foram preenchidas de forma a obter duas condições de compactação: menor densidade e maior densidade do solo. O conteúdo de água nos solos, inicialmente elevado até o ponto de saturação, foi monitorado diariamente por meio de um medidor eletrônico tipo TDR (Profile Probe PR2 acoplado ao Moisture Meter HH2). A resistência do solo à penetração foi mensurada por meio de um penetrômetro de impacto adaptado para vaso. Os pares de dados entre a resistência do solo à penetração e o conteúdo de água foram ajustados, e as CRP foram submetidas ao teste de significância. A relação entre a resistência do solo à penetração e o conteúdo de água foi descrita pelo modelo exponencial decrescente: $R P=A+B e^{-\frac{U g}{C}}$, em que $R P$ representa a resistência do solo à penetração $(\mathrm{MPa}) ; \boldsymbol{U} g$ é o conteúdo de água $\left(\mathrm{kg} \mathrm{kg}^{-1}\right)$; e $A, B$ e $C$ são os coeficientes da equação. Foram obtidos coeficientes de determinação que variaram de 0,79 a 0,96 .
\end{abstract}

Termos de indexação: compactação, conteúdo de água, qualidade física do solo.

\footnotetext{
(1) Parte da Dissertação de Mestrado do primeiro autor. Recebido para publicação em abril de 2008 e aprovado em outubro de 2008.

${ }^{(2)}$ Doutoranda do Departamento de Solos e Adubos da Faculdade de Ciências Agrárias e Veterinárias, Universidade Estadual Paulista - FCAV/UNESP. Via de Acesso Professor Paulo Donato Castellane s/n, CEP 14884-900 Jaboticabal (SP). Bolsista da FAPESP. E-mail: cinarax@yahoo.com.br

${ }^{(3)}$ Professor do Departamento de Solos e Adubos, FCAV/UNESP. Bolsista CNPq. E-mail: jfcentur@fcav.unesp.br

(4) Pós-Doutorando do Departamento de Solos e Adubos, FCAV/UNESP. Bolsista da FAPESP. E-mail: onafreddi@fcav.unesp.br

(5) Doutorando do Departamento de Solos e Adubos, FCAV/UNESP. Bolsista Capes. E-mail: jrfalqueto@yahoo.com.br

${ }^{(6)}$ Professor Titular do Departamento de Ciências Exatas, FCAV/UNESP. Bolsista CNPq. E-mail: jcbarbosa@fcav.unesp.br
} 


\title{
SUMMARY: PEDOTRANSFER FUNCTIONS FOR SOIL RESISTANCE TO PENETRATIONCURVE
}

\begin{abstract}
The estimate soil resistance to penetration curves (RPC) based on readily obtained variables such as water content, is a useful not only for the evaluation of soil compaction, but also to simplify the interpretation of soil resistance to penetration under different field conditions. The objective of this study was to estimate the RPC in soils of different textures and bulk densities based on data obtained with an impact penetrometer. The experiment was carried out at the Faculdade de Ciências Agrárias e Veterinárias (UNESP), Jaboticabal, SP. Four soil classes were used: Entissol, Alfissol, Oxisol sandy loam and clayey Oxisol, which were sampled at a depth of $0-0.20 \mathrm{~m}$. The PVC columns (diameter $0.25 \mathrm{~m}$, height $0.6 \mathrm{~m}$ ) were filled to represent non compacted and compacted soil. The soil water content, initially elevated to the saturation point, was monitored daily with a TDR device (Profile Probe PR2 connected to a HH2 Moisture Meter). The soil resistance to penetration was measured by an impact penetrometer adapted to be used in the pot. The soil resistance to penetration and water content data pairs were adjusted and the RPC submitted to a significance test. The relationship between the soil resistance to penetration and soil water content was best described by the exponential equation: $R P=A+B e^{-\frac{U g}{C}}$, where $R P$ is the soil resistance to penetration (MPa), $V g$ soil water content $\left(\mathrm{kg} \mathrm{kg}^{-1}\right)$ and $A, B$ and $C$ are the equation parameters. There was a significant relation between soil resistance to penetration and water content and the equations fit the data with coefficients of determination ranging from 0.79 to 0.96 .
\end{abstract}

Index terms: compaction, soil water content, soil physical quality.

\section{INTRODUÇÃO}

Dentre as funções do solo, uma das mais importantes é fornecer ao sistema radicular das plantas um ambiente adequado para o seu crescimento e desenvolvimento. Um ambiente propício é aquele no qual as raízes crescem sem encontrar impedimentos e suprem-se de água e nutrientes em quantidade necessária para que as plantas expressem o seu máximo potencial produtivo. No entanto, nem sempre essas condições são encontradas no campo. Ao contrário, muitas vezes o crescimento radicular encontra-se restrito, devido à impedância mecânica ocasionada pela compactação do solo (Passioura, 2002).

A compactação do solo é fortemente correlacionada com a resistência do solo à penetração, mensurada por meio da penetrometria (Bengough \& Mullins, 1990). Esse método apresenta algumas vantagens por ser rápido, fácil e possuir correlação com o crescimento radicular das plantas (Bengough et al., 2001). Como desvantagem, destaca-se a forte relação de dependência entre a resistência do solo à penetração e o seu conteúdo de água (Bailey et al., 1986). A limitação é que, com a diminuição do conteúdo de água, há um incremento na resistência do solo à penetração (Freddi et al., 2006; Holland, 2006; Lima et al., 2006), o que faz com que haja uma sub ou superestimativa na interpretação dos resultados (Busscher et al., 1997).

A resistência do solo à penetração obtida em diferentes condições de densidade e, ou, conteúdo de água é difícil de ser comparada (Bailey et al., 1986). Além disso, conforme Busscher (1990), diferenças na resistência do solo à penetração, ocasionadas por diferentes tratamentos, podem não ser detectadas devido ao efeito do seu conteúdo de água.

A correção dos dados de resistência do solo à penetração no mesmo conteúdo de água pode reduzir problemas de interpretação de resultados obtidos em diversas condições de campo e sistemas de manejo (Busscher et al., 1997). Nesse sentido, pesquisadores têm procurado desenvolver funções de pedotransferência que traduzam essa relação.

Uma equação clássica, muito utilizada na estimativa da resistência à penetração dos solos do Brasil (Tormena et al., 1998; Imhoff et al., 2000; Beutler et al., 2004; Klein \& Câmara, 2007; Lima et al., 2007), foi proposta por Busscher (1990). Avaliando a capacidade de 10 diferentes funções em descrever a resistência do solo à penetração, esse pesquisador identificou uma equação não linear que se ajustou aos dados com elevados coeficientes de determinação. No entanto, a resistência do solo à penetração é determinada não só a partir do conteúdo de água, mas também da densidade do solo. To \& Kay (2005) descreveram a variação da resistência do solo à penetração, em função do seu potencial de água, e os parâmetros da equação estimados a partir do teor de argila e de matéria orgânica e da densidade do solo. Contudo, Dexter et al. (2007) propuseram, para essa relação, uma equação composta por dois termos principais: o primeiro está baseado no grau de compactação e na estruturação do solo e se refere ao índice S proposto por Dexter (2004a,b,c), e o segundo é atribuído ao conteúdo de água no solo. Já Busscher 
et al. (1997) propuseram três diferentes equações exponenciais fundamentadas apenas no conteúdo gravimétrico de água, porém ajustadas a partir de dados coletados em solos desenvolvidos sob influência de clima temperado. Dias Júnior et al. (2004) também relacionaram a resistência do solo à penetração e o conteúdo gravimétrico de água no solo, a partir de amostras coletadas em um Argissolo Amarelo e em um Plintossolo, por meio de modelos lineares.

Assim, modelos matemáticos que representam a dependência da resistência do solo à penetração e seu conteúdo de água ajudam a entender a relação entre essas duas variáveis, favorecendo o entendimento das propriedades mecânicas do solo que governam a compactação. Além disso, representam uma importante ferramenta para a padronização da resistência do solo à penetração quando obtida em diferentes condições de água no solo, o que contribui para a identificação das condições físicas do solo limitantes à produtividade das culturas. Logo, o objetivo deste estudo foi obter funções de pedotransferência que expliquem a relação entre a resistência do solo à penetração e o seu conteúdo de água a partir de dados obtidos com o penetrômetro de impacto, em solos de diferentes granulometrias e densidades.

\section{MATERIAL E MÉTODOS}

O experimento foi realizado no laboratório de Pedologia do Departamento de Solos e Adubos, na Faculdade de Ciências Agrárias e Veterinárias (UNESP), Jaboticabal, SP. As classes de solos utilizadas foram: Neossolo Quartzarênico (RQ), Argissolo Vermelho-Amarelo (PVA), Latossolo Vermelho distrófico (LVd) e Latossolo Vermelho acriférrico (LVwf), classificados segundo Embrapa (2006).

Amostras de cada classe de solo foram coletadas na camada de 0-0,20 m. A composição granulométrica foi determinada pelo método da pipeta (Gee \& Bauder, 1986), o conteúdo de matéria orgânica, quantificado de acordo com Raij et al. (1987), e os óxidos de Fe e Al foram extraídos por ataque sulfúrico (Camargo et al., 1986) (Quadro 1).
Colunas de PVC, com 0,25 m de diâmetro e 0,60 m de altura, foram preenchidas com amostras de cada solo (seco ao ar e passado em peneira de $4 \mathrm{~mm}$ ), de modo a obter duas condições de compactação: menor densidade (D1) e maior densidade do solo (D2). Para a D1, as colunas foram apenas preenchidas com os solos, sem compactação. A partir dos resultados das densidades desses solos, um valor $20 \%$ maior de densidade foi estimado, a partir do qual foi determinada uma massa, a fim de se proceder à compactação do solo para a D2. Para que o solo se acomodasse dentro do volume predeterminado, foi colocado aos poucos dentro das colunas, batendo-se 10 vezes sobre lençol de borracha de $5 \mathrm{~mm}$ de espessura, com distância de queda de mais ou menos $10 \mathrm{~cm}$. Repetiu-se essa operação por mais duas vezes, até que o nível do solo ficasse nivelado com o traço do aferimento do volume.

Inicialmente, o conteúdo de água no solo foi elevado até a saturação, a partir da qual foram obtidas leituras da resistência do solo à penetração $(\mathrm{RP})$ em diferentes conteúdos de água. A RP foi quantificada nas camadas de $0-0,10,0,10-0,20$ e $0,20-0,30 \mathrm{~m}$, por meio de um penetrômetro de impacto (Stolf, 1991), adaptado para vaso, com as seguintes características: área da base com $1,25 \mathrm{~cm}^{2}$; altura de queda variando de 0,05 a $0,40 \mathrm{~m}$; massa do êmbolo, ou seja, massa que provoca o impacto, igual a $1,02 \mathrm{~kg}$; e massa dos demais componentes do penetrômetro, sem o êmbolo, de $0,57 \mathrm{~kg}$. A transformação dos valores da penetração da haste do aparelho no solo (impactos $\mathrm{cm}^{-1}$ ) em RP (MPa) foi feita segundo Stolf (1991).

O conteúdo volumétrico de água foi avaliado nas mesmas camadas, por meio de um medidor eletrônico composto pelo Profile Probe PR2 acoplado ao Moisture Meter HH2 (TDR), e convertido à base gravimétrica (Ug) por meio da densidade do solo.

Foram coletadas ainda amostras indeformadas por meio de cilindros de 53,16 mL, que foram saturadas e submetidas às tensões de 0,$001 ; 0,006 ; 0,01 ; 0,033$; 0,06; 0,1; e 0,3 MPa, em câmaras de Richards (Klute, 1986). Nessas amostras, foram determinados o conteúdo de água retido em cada tensão (Klute, 1986); a densidade do solo, segundo Blake \& Hartge (1986); a microporosidade, por secagem (tensão de 0,006 MPa), em câmaras de pressão de Richards com placa porosa

Quadro 1. Propriedades físicas e químicas dos solos

\begin{tabular}{|c|c|c|c|c|c|c|c|c|c|c|}
\hline Solo & AMG & AG & AM & AF & AMF & Silte & Argila & MO & $\mathrm{Fe}_{2} \mathrm{O}_{3}$ & $\mathrm{Al}_{2} \mathrm{O}_{3}$ \\
\hline & & & & $g$ & & & & 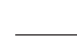 & dag $\mathrm{kg}^{-1}$ & 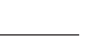 \\
\hline $\mathrm{RQ}$ & 0 & 23 & 233 & 477 & 117 & 33 & 117 & 0,5 & 1,2 & 3,5 \\
\hline PVA & 0 & 110 & 310 & 290 & 143 & 57 & 90 & 1,8 & 1,2 & 2,5 \\
\hline LVd & 0 & 43 & 220 & 320 & 63 & 47 & 307 & 1,4 & 5,3 & 9,5 \\
\hline LVwf & 0 & 10 & 37 & 97 & 40 & 61 & 755 & 4,0 & 24,4 & 22,3 \\
\hline
\end{tabular}

AMG: areia muito grossa (2,0-1,0 mm); AG: areia grossa (0,5-1,0 mm); AM: areia média $(0,25-0,50 \mathrm{~mm})$; AF: areia fina $(0,105-$ $0,250 \mathrm{~mm})$; AMF: areia muito fina $(<0,105 \mathrm{~mm})$; MO: matéria orgânica. 
(Klute, 1986); e a porosidade total, conforme Danielson \& Sutherland (1986). A macroporosidade foi calculada como a diferença entre a porosidade total e a microporosidade (Quadro 2).

As curvas de retenção de água foram ajustadas pelo modelo proposto por van Genuchten (1980), a partir do qual foi obtido o conteúdo de água retido na capacidade de campo (tensão de 0,006 MPa) e no ponto de murcha permanente (tensão de 1,5 MPa) (Quadro 3).
A relação entre a resistência do solo à penetração $\mathrm{e}$ o conteúdo de água foi descrita pelo modelo exponencial decrescente, representado pela equação: $R P=A+B e^{-\frac{U g}{C} \text {; }}$ em que $R P$ representa a resistência do solo à penetração; $U g$ é o conteúdo gravimétrico de água; e $A, B$ e $C$ são os coeficientes da equação.

Para o ajuste entre a RP e o Ug utilizou-se o software Microcal Origin 6.0. Dessa forma, obtiveramse as curvas de resistência do solo à penetração (CRP),

Quadro 2. Densidade, porosidade total, macroporosidade e microporosidade, nas camadas de 0-0,10, 0,100,20 e 0,20-0,30 m, nas diferentes intensidades de compactação

\begin{tabular}{|c|c|c|c|c|c|c|}
\hline \multirow{3}{*}{ Solo } & \multicolumn{6}{|c|}{ Camada } \\
\hline & \multicolumn{2}{|c|}{$0-0,10 \mathrm{~m}$} & \multicolumn{2}{|c|}{$0,10-0,20 \mathrm{~m}$} & \multicolumn{2}{|c|}{$0,20-0,30 \mathrm{~m}$} \\
\hline & D1 & D2 & D1 & D2 & D1 & $\overline{\mathrm{D} 2}$ \\
\hline & \multicolumn{6}{|c|}{ Densidade do solo $\left(\mathrm{Mg} \mathrm{m}^{-3}\right)$} \\
\hline $\mathrm{RQ}$ & 1,49 & 1,77 & 1,55 & 1,70 & 1,52 & 1,74 \\
\hline PVA & 1,37 & 1,60 & 1,34 & 1,53 & 1,32 & 1,57 \\
\hline $\mathrm{LVd}$ & 1,32 & 1,58 & 1,30 & 1,54 & 1,27 & 1,60 \\
\hline \multirow[t]{2}{*}{ LVwf } & 1,13 & 1,29 & 1,12 & 1,33 & 1,10 & 1,32 \\
\hline & \multicolumn{6}{|c|}{ Porosidade total $\left(\mathrm{m}^{3} \mathrm{~m}^{-3}\right)$} \\
\hline $\mathrm{RQ}$ & 0,40 & 0,36 & 0,39 & 0,38 & 0,42 & 0,37 \\
\hline PVA & 0,50 & 0,41 & 0,48 & 0,46 & 0,48 & 0,45 \\
\hline $\mathrm{LVd}$ & 0,49 & 0,44 & 0,52 & 0,47 & 0,52 & 0,45 \\
\hline \multirow[t]{2}{*}{ LVwf } & 0,61 & 0,57 & 0,61 & 0,56 & 0,63 & 0,58 \\
\hline & \multicolumn{6}{|c|}{ Macroporosidade $\left(\mathrm{m}^{3} \mathrm{~m}^{-3}\right)$} \\
\hline $\mathrm{RQ}$ & 0,22 & 0,12 & 0,20 & 0,16 & 0,25 & 0,14 \\
\hline PVA & 0,31 & 0,18 & 0,30 & 0,26 & 0,31 & 0,21 \\
\hline LVd & 0,21 & 0,14 & 0,23 & 0,19 & 0,25 & 0,15 \\
\hline \multirow[t]{2}{*}{ LVwf } & 0,13 & 0,09 & 0,13 & 0,06 & 0,17 & 0,08 \\
\hline & \multicolumn{6}{|c|}{ Microporosidade $\left(\mathrm{m}^{3} \mathrm{~m}^{-3}\right.$} \\
\hline $\mathrm{RQ}$ & 0,18 & 0,24 & 0,19 & 0,22 & 0,17 & 0,23 \\
\hline PVA & 0,19 & 0,23 & 0,18 & 0,21 & 0,17 & 0,23 \\
\hline $\mathrm{LVd}$ & 0,28 & 0,30 & 0,29 & 0,28 & 0,27 & 0,31 \\
\hline LVwf & 0,48 & 0,48 & 0,48 & 0,50 & 0,46 & 0,50 \\
\hline
\end{tabular}

D1: menor densidade do solo; D2: maior densidade do solo; RQ: Neossolo Quartzarênico; PVA: Argissolo Vermelho-Amarelo; LVd: Latossolo Vermelho distrófico; LVwf: Latossolo Vermelho acriférrico.

Quadro 3. Conteúdo de água na capacidade de campo (CC) e no ponto de murcha permanente (PMP), nas diferentes intensidades de compactação dos solos

\begin{tabular}{cccc}
\hline Solo & Nível de compactação & CC & PMP \\
\cline { 3 - 4 } & & 0,11 & 0,04 \\
RQ & D1 & 0,16 & 0,04 \\
D2 kg-1 & 0,14 & 0,05 \\
PVA & D1 & 0,15 & 0,06 \\
& D2 & 0,20 & 0,10 \\
LV1 & D2 & 0,23 & 0,10 \\
LVwf & D1 & 0,41 & 0,26 \\
& D2 & 0,47 & 0,26
\end{tabular}

RQ: Neossolo Quartzarênico; PVA: Argissolo Vermelho-Amarelo; LVd: Latossolo Vermelho distrófico; LVwf: Latossolo Vermelho acriférrico; D1: menor densidade do solo; D2: maior densidade do solo; CC: capacidade de campo a -0,006 MPa e PMP: ponto de murcha permanente a $-1,5 \mathrm{MPa}$. 
que foram então submetidas ao teste F, utilizando-se o aplicativo Statgraphics Plus 6.0.

\section{RESULTADOS E DISCUSSÃO}

Na figura 1 estão representadas, para cada solo e condição de compactação, as regressões não lineares entre a resistência do solo à penetração $(\mathrm{RP})$ e o conteúdo gravimétrico de água no solo (Ug). Observase que o modelo foi eficiente em explicar as variações da RP, que foram significativamente relacionadas com o Ug em todos os solos nas diferentes densidades. Houve diminuição exponencial da RP com incremento no Ug, como também demonstrado por Rosolem et al. (1999), Imhoff et al. (2000), Freddi et al. (2006), Holland (2006) e Lima et al. (2006). Segundo Larson et al. (1980), esse fato ocorre porque a água atua como "lubrificante" entre as partículas de solo, diminuindo a força de coesão entre elas. Esse processo é favorecido pelo aumento do Ug, ou seja, quanto maior for o $\mathrm{Ug}$, menor será a resistência oferecida pelo solo à penetração das raízes. Conforme Camargo (1983), o alongamento radicular só é possível quando a pressão de crescimento das raízes é maior do que a $\mathrm{RP}$ oferecida pelo solo.

Considerando os elevados coeficientes de determinação das regressões $\left(R^{2} \geq 0,79\right)$, verifica-se que a RP pode ser estimada a partir do Ug. Fidalski \& Tormena (2007) e Lima et al. (2007) obtiveram, para o ajuste dos dados de RP em função dos valores de densidade do solo e de Ug, coeficientes de determinação iguais a 0,87 e 0,83 para um Argissolo Vermelho distrófico latossólico e para um Argissolo Vermelho distrófico arênico, respectivamente. Lima et al. (2006), estudando um Latossolo Vermelho-Amarelo de textura franco-arenosa, obtiveram $R^{2}=0,71$ para essa curva de resistência do solo à penetração (CRP), com a RP mensurada em laboratório por um penetrômetro eletrônico, e $\mathrm{R}^{2}=0,66$, quando a $\mathrm{RP}$ foi determinada em campo, com o auxílio de um penetrômetro estático. Esses pesquisadores concluíram que a maneira de obtenção da RP (campo ou laboratório) não influenciou a CRP.

Neste trabalho, contudo, o modelo não se ajustou aos dados quando foram agrupadas as quatro classes de solos e as diferentes densidades $\left(\mathrm{R}^{2}<0,19\right)$. To \& Kay (2005) obtiveram um $\mathrm{R}^{2}$ igual a 0,47 com o ajuste
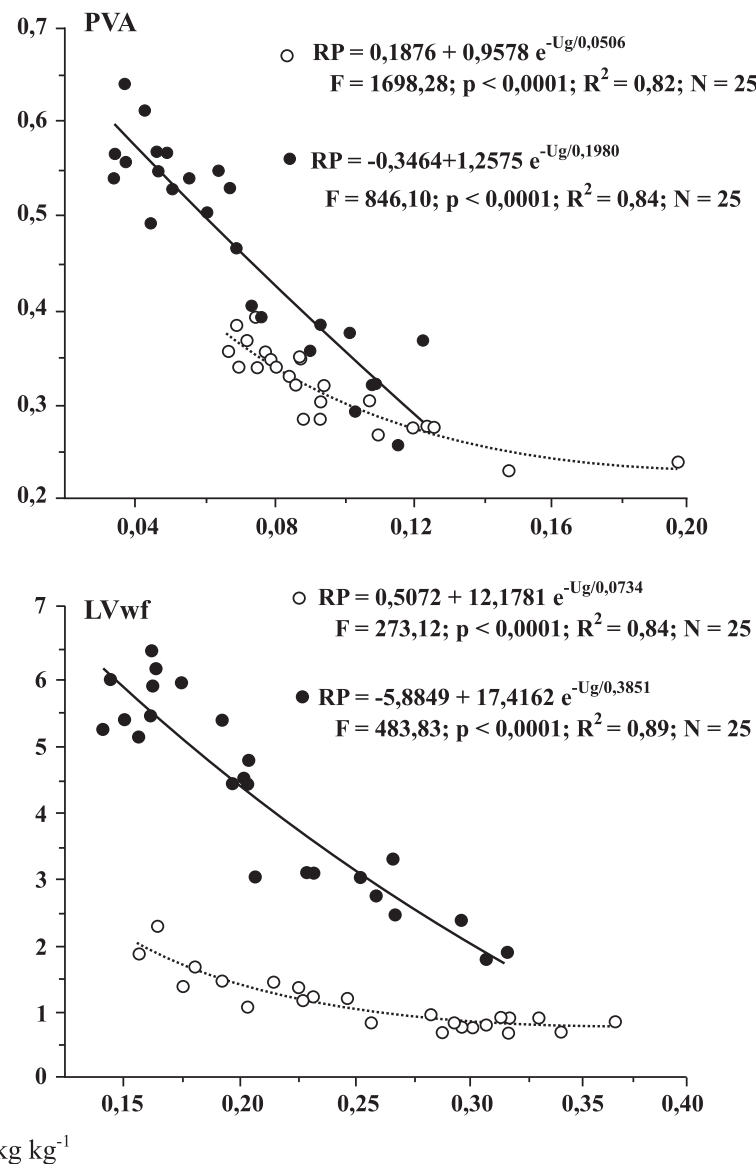

Figura 1. Curvas de resistência dos solos à penetração: Neossolo Quartzarênico (RQ), Argissolo VermelhoAmarelo (PVA), Latossolo Vermelho distrófico (LVd) e Latossolo Vermelho acriférrico (LVwf), nas diferentes intensidades de compactação (OD1 e @D2). 
dos dados realizado a partir de solos de várias classes texturais. Entretanto, o valor do $\mathrm{R}^{2}$ aumentou a partir do ajuste de dados obtidos em solos de classes texturais semelhantes, num trabalho desenvolvido em laboratório a partir de amostras indeformadas coletadas em campo. Essa diferenciação, conforme o teor de argila, também foi observada por Rosolem et al. (1999). Busscher et al. (1997) e Sojka et al. (2001) não alcançaram ajustes satisfatórios quando a CRP foi traçada a partir de dados obtidos em diferentes sistemas de manejo do solo, indicando que apenas uma única equação pode não se ajustar a esses dados.

$\mathrm{O}$ coeficiente A do modelo testado neste trabalho representa a assíntota horizontal da curva (em que $\mathrm{RP}=\mathrm{A}$ ). É o menor valor de $\mathrm{RP}$ que se mantém constante a partir de um determinado Ug. Para todos os solos, nas diferentes densidades, esse valor se dá em Ug a partir da capacidade de campo (tensão $=0,006 \mathrm{MPa}$ ). Esse processo ocorre devido às forças de coesão e adesão que atuam nos solos. A coesão se dá pela atração entre os componentes do solo. A adesão, por sua vez, é resultante da tensão superficial, dependendo da presença de água, sendo inversamente relacionada com o diâmetro capilar, atingindo o máximo de expressão quando o menisco apresenta o máximo de curvatura. Assim, a adesão é mínima ou praticamente inexistente quando o solo está seco. Nesse momento, apenas as forças de coesão estão atuando. Conforme vai aumentando o Ug, a coesão desaparece e há um aumento gradual da adesão, até que um menisco de maior curvatura se forme. A partir desse ponto, acréscimos no Ug diminuem progressivamente a tensão superficial, ou seja, a adesão chega a ser praticamente nula no solo saturado (Oliveira, 2001). Isso explica, em grande parte, o comportamento da RP junto à assíntota horizontal da CRP. Segundo Lima et al. (2007), quanto mais próximo da capacidade de campo estiver o Ug, maiores valores de densidade do solo são possíveis sem que seja superada a RP crítica ao crescimento radicular. Dessa forma, muitos pesquisadores têm realizado as medidas de RP com o Ug próximo à capacidade de campo (Arshad et al., 1996; Beutler et al., 2002; Freddi et al., 2006), pois, segundo Arshad et al. (1996), nessa situação é obtida boa correlação desse atributo com o crescimento radicular das plantas.

O quadro 4 contém a análise descritiva dos dados de RP e Ug para todos os solos nas densidades analisadas. Os altos coeficientes de variação representam a amplitude de variação do Ug e a influência desse atributo nos valores de RP. Também Freddi et al. (2006) e Lima et al. (2006) obtiveram elevados coeficientes de variação ao traçar essa relação, salientando a importância da padronização do Ug na determinação e interpretação da RP.

Os solos de textura arenosa apresentaram valores máximos observados de RP de 2,10 MPa para o RQ e 0,65 MPa para o PVA (Quadro 4), mesmo estando em baixo Ug e maior densidade. Souza et al. (2005) encontraram em um Neossolo Quartzarênico sob Cerrado $\mathrm{RP}$ igual a $0,50 \mathrm{MPa}$, quando ele apresentava Ug igual a $0,09 \mathrm{~kg} \mathrm{~kg}^{-1}$. Silva et al. (2005) também obtiveram valores baixos de RP em um Argissolo

Quadro 4. Média, desvio-padrão, valores (mínimo e máximo) e coeficiente de variação (CV) das variáveis analisadas para o Neossolo Quartzarênico (RQ), Argissolo Vermelho-Amarelo (PVA), Latossolo Vermelho distrófico (LVd) e para o Latossolo Vermelho acriférrico (LVwf), em diferentes intensidades de compactação

\begin{tabular}{|c|c|c|c|c|c|c|c|}
\hline Solo & Nível de compactação & Variável & Média & Desvio-padrão & Mínimo & Máximo & $\mathrm{CV}$ \\
\hline \multirow{4}{*}{ RQ } & \multirow{2}{*}{ D1 } & $\mathrm{Ug}$ & 0,12 & 0,03 & 0,07 & 0,18 & 26,13 \\
\hline & & $\mathrm{RP}$ & 0,41 & 0,08 & 0,26 & 0,61 & 19,31 \\
\hline & \multirow[t]{2}{*}{ D2 } & $\mathrm{Ug}$ & 0,06 & 0,03 & 0,02 & 0,12 & 23,97 \\
\hline & & $\mathrm{RP}$ & 0,75 & 0,50 & 0,27 & 2,10 & 16,01 \\
\hline \multirow{4}{*}{ PVA } & \multirow{2}{*}{ D1 } & $\mathrm{Ug}$ & 0,12 & 0,03 & 0,09 & 0,21 & 40,64 \\
\hline & & $\mathrm{RP}$ & 0,29 & 0,05 & 0,20 & 0,38 & 71,97 \\
\hline & \multirow{2}{*}{ D2 } & $\mathrm{Ug}$ & 0,09 & 0,03 & 0,06 & 0,14 & 23,38 \\
\hline & & $\mathrm{RP}$ & 0,47 & 0,12 & 0,23 & 0,65 & 44,26 \\
\hline \multirow{4}{*}{ LVd } & \multirow{2}{*}{ D1 } & $\mathrm{Ug}$ & 0,13 & 0,05 & 0,05 & 0,28 & 42,06 \\
\hline & & $\mathrm{RP}$ & 0,67 & 0,48 & 0,25 & 2,07 & 66,19 \\
\hline & \multirow{2}{*}{ D2 } & $\mathrm{Ug}$ & 0,10 & 0,03 & 0,06 & 0,15 & 29,33 \\
\hline & & $\mathrm{RP}$ & 2,06 & 1,44 & 0,38 & 5,27 & 24,61 \\
\hline \multirow{4}{*}{ LVwf } & \multirow{2}{*}{ D1 } & $\mathrm{Ug}$ & 0,27 & 0,06 & 0,16 & 0,38 & 30,10 \\
\hline & & $\mathrm{RP}$ & 0,94 & 0,42 & 0,50 & 2,16 & 69,90 \\
\hline & \multirow{2}{*}{ D2 } & $\mathrm{Ug}$ & 0,22 & 0,06 & 0,14 & 0,33 & 25,59 \\
\hline & & $\mathrm{RP}$ & 4,11 & 1,50 & 1,65 & 6,36 & 36,43 \\
\hline
\end{tabular}

Ug: conteúdo gravimétrico de água $\left(\mathrm{kg} \mathrm{kg}^{-1}\right)$; RP: resistência do solo à penetração $(\mathrm{MPa})$; D1: menor densidade do solo; D2: maior densidade do solo. 
Vermelho textura média, que variaram de 0,22 a $0,75 \mathrm{MPa}$, em Ug de 0,22 a 0,17 kg kg-1, em diferentes sistemas de manejo e camadas analisadas. O maior valor de RP encontrado por Abreu et al. (2004), em um Argissolo Vermelho-Amarelo de textura franco-arenosa, foi de 1,60 MPa no tratamento sob cultivo mínimo, com Ug de 0,13 kg kg-1. Contudo, esses autores afirmam que a RP é mais sensível em detectar a compactação do que a densidade ou a porosidade do solo.

Todavia, tanto o LVd quanto o LVwf apresentaram valores maiores de RP, quando em maior densidade e em valores de Ug mais baixos, comparados aos solos mais arenosos (Quadro 4). Para o LVd, o maior valor observado de RP foi de 5,27 MPa, e, para o LVwf, este valor foi de 6,36 MPa. Tormena et al. (1998) constataram que valores de RP restritivos ao crescimento das plantas podem ser encontrados sob baixos valores de densidade do solo, devido ao baixo Ug, num Latossolo Vermelho muito argiloso (800 $\mathrm{g} \mathrm{kg}^{-1}$ de argila) sob sistema de semeadura direta. Valores em torno de 4,0 e 2,0 MPa de RP foram encontrados por Tormena et al. (2002) em um Latossolo Vermelho textura média, relativamente seco, com Ug de 0,15 e $0,14 \mathrm{~kg} \mathrm{~kg}^{-1}$, sob semeadura direta e sob cultivo convencional, respectivamente. No entanto, nesse mesmo solo, só que na capacidade de campo $\left(0,17 \mathrm{~kg} \mathrm{~kg}^{-1}\right)$, houve diminuição da $\mathrm{RP}$ para $2,3 \mathrm{MPa}$ no sistema de semeadura direta e para $1,0 \mathrm{MPa}$ aproximadamente no preparo convencional. Esses pesquisadores concluíram que no solo mais úmido os valores de RP podem ser considerados não impeditivos para o preparo convencional e pouco impeditivos para o plantio direto e que, com o secamento do solo, os valores podem atingir níveis altamente impeditivos, especialmente sob o sistema de semeadura direta. Estudando classe de solo semelhante, Araújo et al. (2004) também constataram que a RP, quantificada sob mata nativa, somente atinge valores considerados críticos ao crescimento das raízes em Ug muito baixo $\left(<0,10 \mathrm{~kg} \mathrm{~kg}^{-1}\right)$ e em densidade elevada (acima de $1,56 \mathrm{Mg} \mathrm{m}^{-3}$ ). Esses pesquisadores verificaram que os valores de RP foram muito semelhantes tanto para o solo sob mata quanto para o solo sob cultivo, mesmo que a menor densidade nesse sistema corresponda à maior densidade do solo sob mata, constatando a grande influência da água na RP.

Os resultados comprovam a grande influência do Ug na determinação da RP. Por esse motivo, e também por falta de métodos adequados, muitos, pesquisadores preconizam que a determinação da $R P$ deve ser realizada com o Ug próximo à capacidade de campo, visto que, a partir desse ponto (tensão = 0,006 MPa), essa relação de dependência é menor. A correção dos valores de RP obtidos em diferentes Ug pode reduzir ou eliminar o efeito desse atributo na determinação e interpretação dos resultados obtidos. Assim, o aprimoramento das técnicas de obtenção dos resultados de RP possibilitará não só a determinação da impedância mecânica crítica ao crescimento e desenvolvimento das plantas, como também a quantificação do impacto das práticas de manejo na compactação do solo.

Pesquisas complementares serão necessárias para a validação das funções de pedotransferência elaboradas, a fim de que venham a ser testadas em campo.

\section{CONCLUSÕES}

1. A relação entre a resistência do solo à penetração e o conteúdo de água foi descrita pelo modelo exponencial decrescente, representado pela equação: $R P=A+B e^{-\frac{U g}{C}}$, em que $R P$ representa a resistência do solo à penetração (MPa), Ugé o conteúdo gravimétrico de água $\left(\mathrm{kg} \mathrm{kg}^{-1}\right)$ e $A, B$ e $C$ são os coeficientes da equação.

2. A correção dos dados de resistência do solo à penetração pode carecer de várias equações baseadas em diferentes condições de granulometria e densidade.

\section{AGRADECIMENTO}

À Fundação de Amparo à Pesquisa do Estado de São Paulo - FAPESP, pela concessão da bolsa de estudos ao primeiro autor.

\section{LITERATURA CITADA}

ABREU, S.L.; REICHERT, J.M. \& REINERT, D.J. Escarificação mecânica e biológica para a redução da compactação em Argissolo franco-arenoso sob plantio direto. R. Bras. Ci. Solo, 28:519-531, 2004.

ARAÚJO, M.A.; TORMENA, C.A. \& SILVA, A.P. Propriedades físicas de um Latossolo Vermelho distrófico cultivado e sob mata nativa. R. Bras. Ci. Solo, 28:337-345, 2004.

ARSHAD, M.A.; LOWERY, B. \& GROSSMAN, B. Physical tests for monitoring soil quality. In: DORAN, J.W. \& JONES, A.J. eds. Methods for assessing soil quality. Madison, Soil Science Society of America, 1996. p.123141. (SSSA Special Publication, 49)

BAILEY, A.C.; JOHNSON, C.E. \& SCHAFER, R.L. A model for agricultural soil compaction. J. Agric. Eng. Res., 33:257-262, 1986

BENGOUGH, A.G. \& MULLINS, C.E. Mechanical impedance to root growth: A review of experimental techniques and root growth responses. J. Soil Sci., 41:341-358, 1990.

BENGOUGH, A.G.; CAMPBELL, D.J. \& O'SULLIVAN, M.F. Penetrometer techiniques in relation to soil compaction and root growth. In: SMITH, K.A. \& MULLINS, C.E., eds. Soil and environment analysis: Physical methods. 2.ed. New York, Marcel Decher, 2001. p.377-403. 
BEUTLER, A.N.; CENTURION, J.F. \& SILVA, A.P. Intervalo hídrico ótimo e a produção de soja e arroz em dois Latossolos. Irriga, 9:181-192, 2004.

BEUTLER, A.N.; CENTURION, J.F.; SOUZA, Z.M. \& SILVA, L.M. Utilização dos penetrômetros de impacto e de anel dinamométrico em Latossolos. Eng. Agríc., 22:191-199, 2002.

BLAKE, G.R. \& HARTGE, K.H. Bulk density. In: KLUTE, A. ed. Methods of soil analysis: Physical and mineralogical methods. 2.ed. Madison, American Society of Agronomy, 1986. Part 1. p.363-375.

BUSSCHER, W.J. Ajustment of flatipped penetrometer resistance data to a common water content. Trans. Am. Soc. Agric. Eng., 33:519-523, 1990.

BUSSCHER, W.J.; BAUER, P.J.; CAMP, C.R. \& SOJKA, R.E. Correction of cone index for soil water content differences in a Coastal Plain soil. Soil Till. Res., 43:205-217, 1997.

CAMARGO, O.A. Compactação do solo e desenvolvimento de plantas. Campinas, Fundação Cargill, 1983. 44p.

CAMARGO, O.A.; MONIZ, A.C.; JORGE, J.A. \& VALADARES, J.M.A.S. Métodos de análise química, mineralógica e física de solos do IAC. Campinas, Instituto Agronômico de Campinas, 1986. 94p. (Boletim Técnico, 106)

DANIELson, R.E. \& SUTHERLAND, P.L. Porosity. In: KLUTE, A., ed. Methods of soil analysis: Physical and mineralogical methods. 2.ed. Madison, American Society of Agronomy, 1986. Part 1. p.443-461.

DEXTER, A.R. Soil physical quality. Part I. Theory, effects of soil texture, density, and organic matter, and effects on root growth. Geoderma, 120:201-214, 2004a.

DEXTER, A.R. Soil physical quality. Part II. Friability, tillage, tilth and hard-setting. Geoderma, 120:215-226, 2004b.

DEXTER, A.R. Soil physical quality. Part III. Unsaturated hydraulic conductivity and general conclusions about Stheory. Geoderma, 120:227-239, 2004c.

DEXTER, A.R.; CZYZ, E.A. \& GATE, O.P. A method for prediction of soil penetration resistance. Soil Till. Res., 93:412-419, 2007.

DIAS JÚNIOR, M.S.; SILVA, A.R.; FONSECA, S. \& LEITE, F.P. Método alternativo de avaliação da pressão de preconsolidação por meio de um penetrômetro. R. Bras. Ci. Solo, 28:805-810, 2004.

EMPRESA BRASILEIRA DE PESQUISA AGROPECUÁRIA EMBRAPA. Centro Nacional de Pesquisa de Solos. Sistema brasileiro de classificação de solos. Rio de Janeiro, 2006. 306p.

FIDALSKI, J. \& TORMENA, C.A. Funções de pedotransferência para as curvas de retenção de água e de resistência do solo à penetração em sistemas de manejo com plantas de cobertura permanente em citros. Ci. Rural, 37:1316-1322, 2007.
FREDDI, O.S.; CARVALHO, M.P.; VERONESI JÚNIOR, V. \& CARVALHO, G.J. Produtividade do milho relacionada com a resistência mecânica à penetração do solo sob preparo convencional. Eng. Agríc., 26:113-121, 2006.

GEE, G.W. \& BAUDER, J.W. Particle-size analysis. In: KLUTE, A., ed. Methods of soil analysis: Physical and mineralogical methods. 2.ed. Madison, American Society of Agronomy, 1986. Part. 1. p. 383-411.

HOLLAND, J.E. Changes in soil physical properties under raised bed cropping. Melbourne, Melbourne University, 2006. 274p. (Tese de Doutorado)

IMHOFF, S.; SILVA, A.P. \& TORMENA, C.A. Aplicações da curva de resistência no controle da qualidade física de um solo sob pastagem. Pesq. Agropec. Bras., 35:1493-1500, 2000.

KLEIN, V.A. \& CÂMARA, R.K. Rendimento da soja e intervalo hídrico ótimo em Latossolo Vermelho sob plantio direto escarificado. R. Bras. Ci. Solo, 31:221-227, 2007.

KLUTE, A. Water retention: Laboratory methods. In: KLUTE, A., ed. Methods of soil analysis: Physical and mineralogical methods. 2.ed. Madison, American Society of Agronomy, 1986. Part. 1. p.635-662.

LARSON, W.E.; GUPTA, S.C. \& USECHE, R.A. Compression of agricultural soils from eight soil orders. Soil Sci. Soc. Am. J., 44:450-457, 1980.

LIMA, C.L.R.; REICHERT, J.M.; REINERT, D.J.; SUZUKI, L.E.A.S. \& DALBIANCO, L. Densidade crítica ao crescimento de plantas considerando água disponível e resistência à penetração de um Argissolo Vermelho distrófico arênico. Ci. Rural, 37:1166-1169, 2007.

LIMA, C.L.R.; SILVA, A.P.; IMHOFF, S. \& LEÃO, T.P. Estimativa da capacidade de suporte de carga do solo a partir da avaliação da resistência à penetração. R. Bras. Ci. Solo, 30:217-223, 2006.

OLIVEIRA, J.B. Pedologia aplicada. Jaboticabal, Funep, 2001. $414 \mathrm{p}$.

PASSIOURA, J.B. Soil condictions and plant growth. Plant Cell Environ., 25:311-318, 2002.

RAIJ, B.van; QUAGGIO, J.A.; CANTARELLA, H.; FERREIRA, M.; LOPES, A.S. \& BATAGLIA, O.C. Análise química do solo para fins de fertilidade. Campinas, Fundação Cargill, 1987. 170p.

ROSOLEM, C.A.; FERNANDEZ, E.M.; ANDREOTTI, M. \& CRUSCIOL, C.A.C. Crescimento radicular de plântulas de milho afetado pela resistência do solo à penetração. Pesq. Agropec. Bras., 34:821-828, 1999.

SILVA, M.A.S.; MAFRA, A.L.; ALBUQUERQUE, J.A. \& MIELNICZUK, C.B. Atributos físicos do solo relacionados ao armazenamento de água em um Argissolo Vermelho sob diferentes sistemas de preparo. Ci. Rural, 35:544$552,2005$.

SOJKA, R.E.; BUSSCHER, W.J. \& LEHRSCH, G.A. In situ strength, bulk density and water content relationships of a Durinodic Xeric Haplocalcid soil. Soil Sci., 166:520-529, 2001. 
SOUZA, E.D.; CARNEIRO, M.A.C. \& PAULINO, H.B. Atributos físicos de um Neossolo Quartzarênico e um Latossolo Vermelho sob diferentes sistemas de manejo. Pesq. Agropec. Bras., 40:1135-1139, 2005.

STOLF, R. Teoria e teste experimental de fórmulas de transformação dos dados de penetrômetro de impacto em resistência do solo. R. Bras. Ci. Solo, 15:229-235, 1991.

TO, J. \& KAY, B.D. Variation in penetrometer resistance with soil properties: The contribution of effective stress and implications for pedotransfer functions. Geoderma, 126:261-276, 2005.
TORMENA, C.A.; BARBosA, M.C.; COSTA, A.C.S. \& GONÇALVES, A.C.A. Densidade, porosidade e resistência à penetração em Latossolo cultivado sob diferentes sistemas de preparo do solo. Sci. Agric., 59:795-801, 2002.

TORMENA, C.A.; SILVA, A.P. \& LIBARDI, P.L. Caracterização do intervalo hídrico ótimo de um Latossolo Roxo sob plantio direto. R. Bras. Ci. Solo, 22:573-581, 1998.

Van GENUCHTEN, M.T.A. A closed-form equation for predicting the hydraulic conductivity of unsaturated soils. Soil Sci. Soc. Am. J., 44:892-897, 1980. 